\title{
Wie und wozu braucht es Medizingeschichte?
}

\author{
Das Institut für Medizingeschichte der Universität Bern feierte am 22. November \\ sein 50-jähriges Bestehen. Der Moment wurde als Gelegenheit genutzt, um auf die \\ Vergangenheit zurückzublicken und gleichzeitig die Zukunft in einem öffentlichen \\ Anlass zu konturieren. Im Rahmen einer Podiumsdiskussion stellten sich schliesslich \\ drei Lehrstuhlinhaber der Frage «Wie und wozu Medizingeschichte?».
}

\section{Lina Gafner}

Wissenschaftliche Mitarbeiterin Institut für Medizingeschichte der Universität Bern
Mit der Gründung des Instituts 1963 hat die Medizingeschichte in Bern eine Professionalisierung erfahren und ist seither fest im Lehrplan der ärztlichen Ausbildung verankert. Während sich zuvor historisch interessierte Mediziner nebenamtlich der Geschichte ihres Fachs gewidmet hatten, waren von die wenigen bezahlten Stellen hinausreicht. Medizingeschichte bleibt für viele, die im Institut tätig sind und zu dessen Blühen beitragen, immer auch eine Herzensangelegenheit. Das Fach lebt von seiner Position im disziplinären Ausserhalb, Dazwischen und Darüberhinaus, und dieser Umstand macht die

\section{«Die kritische Reflexion als Teil der ärztlichen Ausbildung ist eine Voraus- setzung dafür, dass sich die Medizin als akademisches Fach verstehen darf.»}

Korrespondenz: Lina Gafner, lic. phil. hist. Universität Bern Bühlstrasse 26 CH-3009 Bern lina.gafner[at]img.unibe.ch nun an habilitierte Medizinhistorikerinnen und -historiker für Forschung, Lehre und damit auch für eine inhaltliche Positionierung des Faches verantwortlich. Dennoch, so ging aus den Vorträgen der Jubiläumsfeier hervor, ist das Institut nach wie vor wesentlich getragen durch ein Engagement, das über

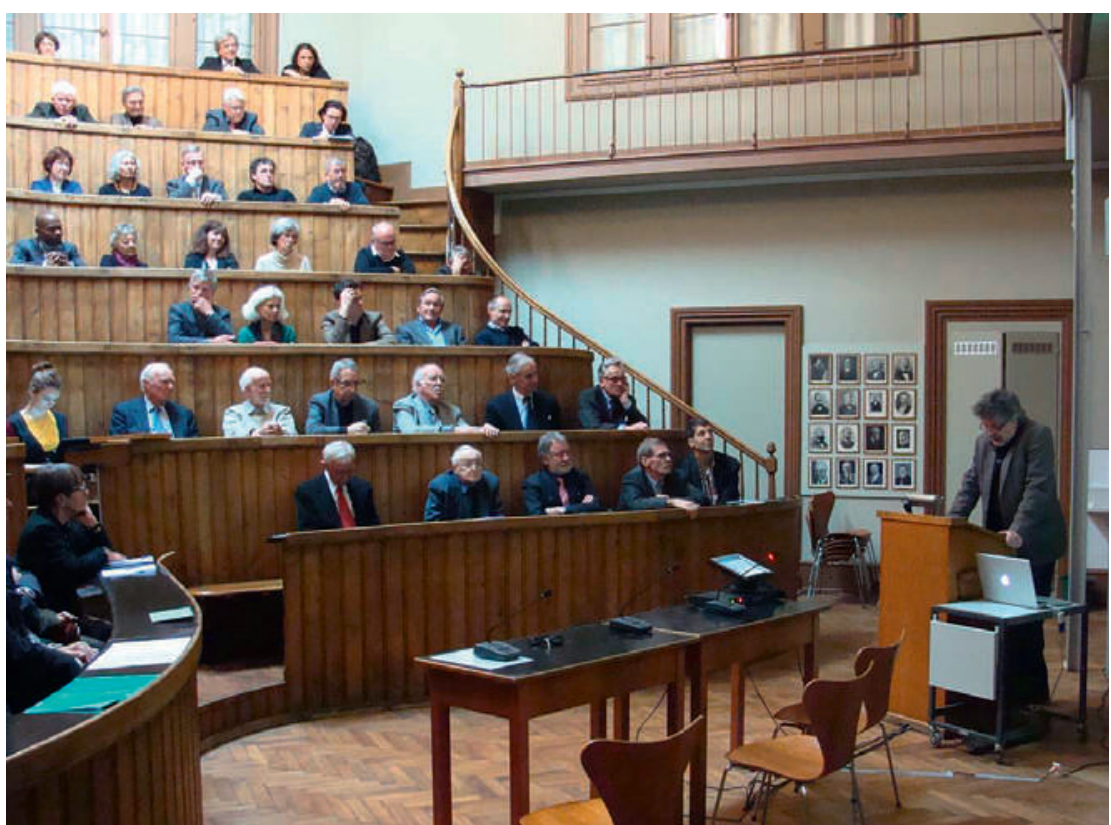

Peter Eggli: Die kritische Reflexion ist wichtiger Teil der ärztlichen Ausbildung.
Frage nach der Aufgabe der Medizingeschichte umso interessanter.

\section{Das Gedächtnis medizinischen Fortschritts?}

Die fortwährende Reflexion und Selbstreflexion, die sich die Medizingeschichte zur Aufgabe macht, sollte auch im Festakt nicht fehlen. Und so wurde der Anlass, der auf grosses Interesse stiess, durch den aktuellen Direktor Prof. Hubert Steinke nicht ohne Vorbehalte angegangen: Die Medizingeschichte habe ein gespaltenes Verhältnis zu Jubiläumsfeiern, denn sie werde gern gerufen, wenn medizinischer Fortschritt und die Kontinuität medizinischer Institutionen gefeiert und durch einen unterhaltsamen Beitrag untermalt sein wollen. Ein durchaus legitimer Auftrag, der dem Anspruch des Fachs allerdings nicht gerecht wird.

Die Fragestellungen der Medizingeschichte haben sich seit ihrer Professionalisierung gewandelt und, entsprechend den wechselnden aktuellen Fragen im Feld der Medizin, aber auch entlang den methodischen Diskussionen in den Geschichtswissenschaften, neu ausgerichtet. Thematisch setzen die drei Institute der Schweiz (Bern, Lausanne und Zürich) unterschiedliche Schwerpunkte. Für das Berner Institut liegt der Fokus der Forschung momentan stark auf der ärztlichen Praxis, der sich zwei verschiedene, vom Nationalfonds finanzierte Forschungsprojekte widmen. Eine der wichtigsten Kapitalien für das Institut ist dabei das interne 


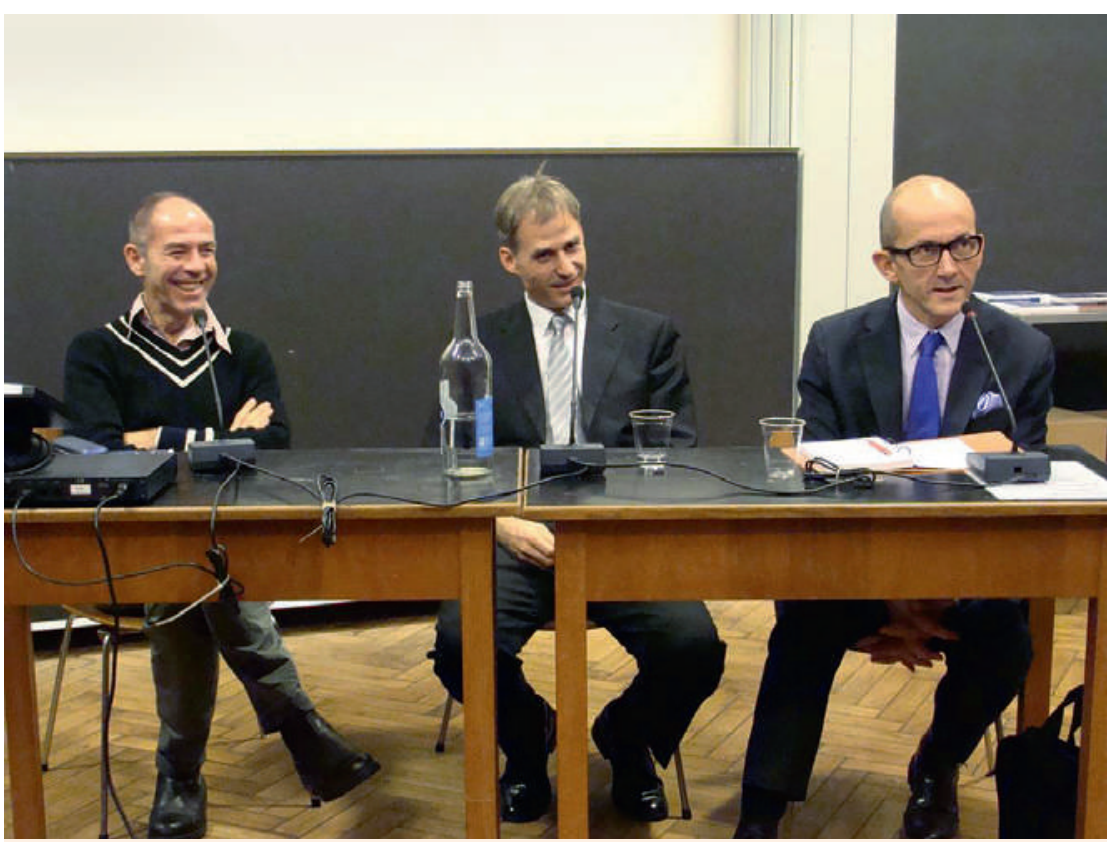

Die Medizinhistoriker Vincent Barras, Hubert Steinke und Flurin Condrau (v. I. n. r.) sind sich einig, dass die Medizingeschichte Medizin von einer Metaebene aus betrachten soll. die Medizingeschichte in der medizinischen Ausbildung als Ort anzubieten, an dem Fragen gestellt werden können, für die ansonsten wenig oder gar kein Raum besteht. Diese Rolle des Fachs ist unumstritten. Wie der Dekan der medizinischen Fakultät Bern Peter Eggli betont, ist gerade die kritische Reflexion als Teil der ärztlichen Ausbildung eine Voraussetzung dafür, dass sich die Medizin als akademisches Fach verstehen darf und nicht einer handwerklichen Berufsausbildung entspricht. Hier leistet die Medizingeschichte einen zentralen Beitrag.

\section{Gute Ärztinnen und Ärzte}

Dennoch stellt sich die Frage, was der Blick zurück den angehenden Ärztinnen und Ärzten denn konkret bieten kann. Die drei Dozenten sind sich darin einig, dass es in ihren Lektionen darum gehen soll, die Medizin von einer Metaebene aus zu betrachten. Ein historischer Zugang, etwa zu der Frage, was einen guten Arzt und eine gute Ärztin auszeichne, legt offen, wie unterschiedlich diese Frage beantwortet worden ist und werden kann. Die Kenntnis der Zusammenhänge historischer Körpervorstellungen und Begrifflichkeiten kann zudem dabei helfen, Patientinnen und Patienten besser zu verstehen, die sich oftmals Krankheitskonzepten bedienen, die nicht der aktuellen akademischen Perspektive entsprechen.

Als weitere Aufgabe sehen die drei Medizinhistoriker, mit den Studierenden das Thema der Wissenschaftlichkeit anzugehen. Als Akademikerinnen und Akademiker sollten diese fähig sein, sich über bestimmte Themen einen Forschungsüberblick zu verschaffen. Eine eigene, kritische Haltung ist dabei nicht bloss gegenüber alten, sondern auch gegenüber neuen Erkenntnissen von Vorteil. Die Studierenden, die sich in der medizinischen Ausbildung insbesondere eine grosse Menge an Wissen aneignen müssen, sollten sich bewusst werden, dass auch dieses Wissen kritisch reflektiert werden sollte. Die Medizingeschichte bietet sich hier an, um im Blick zurück das Gegenwärtige und Neue in ein anderes Licht zu stellen. Diese Aufgabe des Fachs wird auch in Zukunft keine weniger dringliche sein. 